\title{
Novel LRPPRC compound heterozygous mutation in a child with early-onset Leigh syndrome French-Canadian type: case report of an Italian patient
}

Ettore Piro $^{1^{*}}$ D, Gregorio Serra ${ }^{1}$, Vincenzo Antona ${ }^{1}$, Mario Giuffrè ${ }^{1}$, Elisa Giorgio ${ }^{2}$, Fabio Sirchia ${ }^{3}$, Ingrid Anne Mandy Schierz ${ }^{1}$, Alfredo Brusco ${ }^{2}$ and Giovanni Corsello ${ }^{1}$

\begin{abstract}
Background: Mitochondrial diseases, also known as oxidative phosphorylation (OXPHOS) disorders, with a prevalence rate of 1:5000, are the most frequent inherited metabolic diseases. Leigh Syndrome French Canadian type (LSFC), is caused by mutations in the nuclear gene (2p16) leucine-rich pentatricopeptide repeat-containing (LRPPRC). It is an autosomal recessive neurogenetic OXPHOS disorder, phenotypically distinct from other types of Leigh syndrome, with a carrier frequency up to 1:23 and an incidence of 1:2063 in the Saguenay-Lac-St Jean region of Quebec. Recently, LSFC has also been reported outside the French-Canadian population.

Patient presentation: We report a male Italian (Sicilian) child, born preterm at $28+6 / 7$ weeks gestation, carrying a novel LRPPRC compound heterozygous mutation, with facial dysmorphisms, neonatal hypotonia, non-epileptic paroxysmal motor phenomena, and absent sucking-swallowing-breathing coordination requiring, at 4.5 months, a percutaneous endoscopic gastrostomy tube placement. At 5 months brain Magnetic Resonance Imaging showed diffuse cortical atrophy, hypoplasia of corpus callosum, cerebellar vermis hypoplasia, and unfolded hippocampi. Both auditory and visual evoked potentials were pathological. In the following months Video EEG confirmed the persistence of sporadic non epileptic motor phenomena. No episode of metabolic decompensation, acidosis or ketosis, frequently observed in LSFC has been reported. Actually, aged 14 months corrected age for prematurity, the child shows a severe global developmental delay. Metabolic investigations and array Comparative Genomic Hybridization (aCGH) results were normal. Whole-genome sequencing (WGS) found a compound heterozygous mutation in the LRPPRC gene, c.1921-7A > G and c.2056A > G (p.lle686Val), splicing-site and missense variants, inherited from the mother and the father, respectively.

\footnotetext{
* Correspondence: ettore.piro@unipa.it

'Department of Health Promotion, Mother and Child Care, Internal Medicine and Medical Specialties "G. D’Alessandro". University Hospital "P.Giaccone", University of Palermo, Piazza delle Cliniche, 2, 90127 Palermo, Italy Full list of author information is available at the end of the article
}

C C The Author(s). 2020 Open Access This article is licensed under a Creative Commons Attribution 4.0 International License, which permits use, sharing, adaptation, distribution and reproduction in any medium or format, as long as you give appropriate credit to the original author(s) and the source, provide a link to the Creative Commons licence, and indicate if changes were made. The images or other third party material in this article are included in the article's Creative Commons licence, unless indicated otherwise in a credit line to the material. If material is not included in the article's Creative Commons licence and your intended use is not permitted by statutory regulation or exceeds the permitted use, you will need to obtain permission directly from the copyright holder. To view a copy of this licence, visit http://creativecommons.org/licenses/by/4.0/ The Creative Commons Public Domain Dedication waiver (http://creativecommons.org/publicdomain/zero/1.0/) applies to the data made available in this article, unless otherwise stated in a credit line to the data. 


\begin{abstract}
(Continued from previous page)
Conclusions: We first characterized the clinical and molecular features of a novel $L R P P R C$ variant in a male Sicilian child with early onset encephalopathy and psychomotor impairment. Our patient showed a phenotype characterized by a severe neurodevelopmental delay and absence of metabolic decompensation attributable to a probable residual enzymatic activity. LRPPRC is a rare cause of metabolic encephalopathy outside of Québec. Our patient adds to and broaden the spectrum of LSFC phenotypes. WGS analysis is a pivotal genetic test and should be performed in infants and children with hypotonia and developmental delay in whom metabolic investigations and aCGH are normal.
\end{abstract}

Keywords: LSFC, Mitochondrial disease, Hypotonia - developmental delay, Whole-exome sequencing

\section{Introduction}

Leigh syndrome, French-Canadian type (LSFC, MIM\#220 111 ) is a neurogenetic degenerative disorder caused by mutations in the nuclear gene leucine-rich pentatricopeptide repeat-containing (LRPPRC) localized on 2p16. It is phenotypically distinct from other types of Leigh syndrome, for the possible early occurrence of fatally severe metabolic crises [1]. As a result of a founder effect, this rare monogenic autosomal recessive mitochondrial disease is more prevalent in the Saguenay-Lac-St Jean (SLSJ) region of Quebec [2] with a carrier frequency up to 1:23 adults and an incidence of 1:2063 live births [2,3]. LRPPRC is a multifunctional protein belonging to a family of pentatricopeptide repeat (PPR) proteins containing a canonical 35 residue repeat motif that confer an ability to recognize RNA substrates regulating several post-transcriptional processes such as RNA editing, RNA stability or RNA degradation $[4,5]$. Most LSFC patients are homozygous for an $\mathrm{A} 354 \mathrm{~V}$ substitution that causes a decrease in the expression of the LRPPRC protein [6]. Biochemically, LSFC is characterized by a severe cytochrome-c oxidase (COX) deficiency in the brain and liver, while in other tissues, such as kidneys, skeletal muscle, and heart, COX activity is affected to a lesser extent (50$80 \%$ residual activity) $[1,2]$. In recent years, novel diseasecausing mutations have been reported from some unrelated families outside the French-Canadian population [6]. Early onset, from birth to 5 months, has been described as characterized by neonatal neurological findings including beyond hypotonia, transient tachypnea, poor sucking, tremor, along with acute metabolic decompensation including acidosis and ketosis, described as frequently fatal, and stroke-like episodes [1, 7]. In survivors a severe neurological impairment is described including: dystonia, ataxia, dysphagia, strabismus and seizures. Brain imaging has evidenced in some patients, cerebral malformations (cerebellar hypoplasia, gyral abnormalities, and hippocampal abnormalities), leukoencephalopathy, and progressive degenerative involvement of brainstem and basal ganglia $[6,8]$. Consistently dysmorphic cranio-facial features as prominent forehead, anteverted nares, arched eyebrows hypertelorism, with varying degrees of midfacial hypoplasia have been described [2]. Moreover, polysyndactyly, complex congenital heart disease, hypertrophic cardiomyopathy, hypospadias and anteriorly placed anus have been sporadically reported [6].

\section{Patient presentation}

Since our patient was born preterm, in this report we have referred to both a chronological age $(\mathrm{CrA})$ and a corrected age for prematurity $(\mathrm{CA})$, considering the difference of 78 days to reach the 280 days length of full-term pregnancy.

The proband was the second child of healthy and nonconsanguineous Italian parents from Sicily. He was born preterm, at $28+6 / 7$ weeks after a naturally conceived pregnancy, by cesarean section for premature rupture of membranes, chorioamnionitis and fetal distress. Apgar scores were 7 and 7 , at 1 and $5 \mathrm{~min}$, respectively. At birth, his weight was $1360 \mathrm{~g}$ (80th centile), length $40 \mathrm{~cm}$ (86th centile), and occipitofrontal circumference (OFC) $28.4 \mathrm{~cm}$ (89th centile). On physical examination, he showed dysmorphic features: prominent forehead, hypertelorism, broad nasal bridge, anteverted nares, flattened philtrum, thin upper lip, and high-arched palate. Neurological examination revealed, marked generalized hypotonia with presence of deep tendon reflexes, depressed neonatal reflexes, poor spontaneous motility, right exophoria, disconjugated eye movements and weak cry (Fig. 1). Brain ultrasound (US) showed normal corpus callosum and mild dilatation of the left lateral ventricle. He had poor respiratory adaptation with tachypnea needing non-invasive ventilatory support from birth to 3 months CrA (15 days CA). From birth repeated Video-EEG monitoring showed sporadic spontaneous as well as provoked clonic and spontaneous motor paroxysms involving limbs, without concomitant EEG discharge (Fig. 2). He necessitated of frequent oropharyngeal aspirations due to swallowing dysfunction, an early sign of incoordination of sucking-swallowing and breathing. These findings suggested, in relation to failure to thrive and frequent episodes of reflux associated with apnea, to convert to a nasogastric tube feeding regimen until insertion, at 4.5 months $\mathrm{CrA}$, of a percutaneous endoscopic gastrostomy (PEG). At 5 months of $\mathrm{CrA}$, corresponding to 2.5 months $\mathrm{CA}$, a brain MRI revealed a mild diffuse cerebral atrophy, hypoplasia of the corpus callosum, cerebellar vermis hypoplasia (Fig. 3) and bilateral incomplete hippocampal inversion (Fig. 4). Visual evoked 


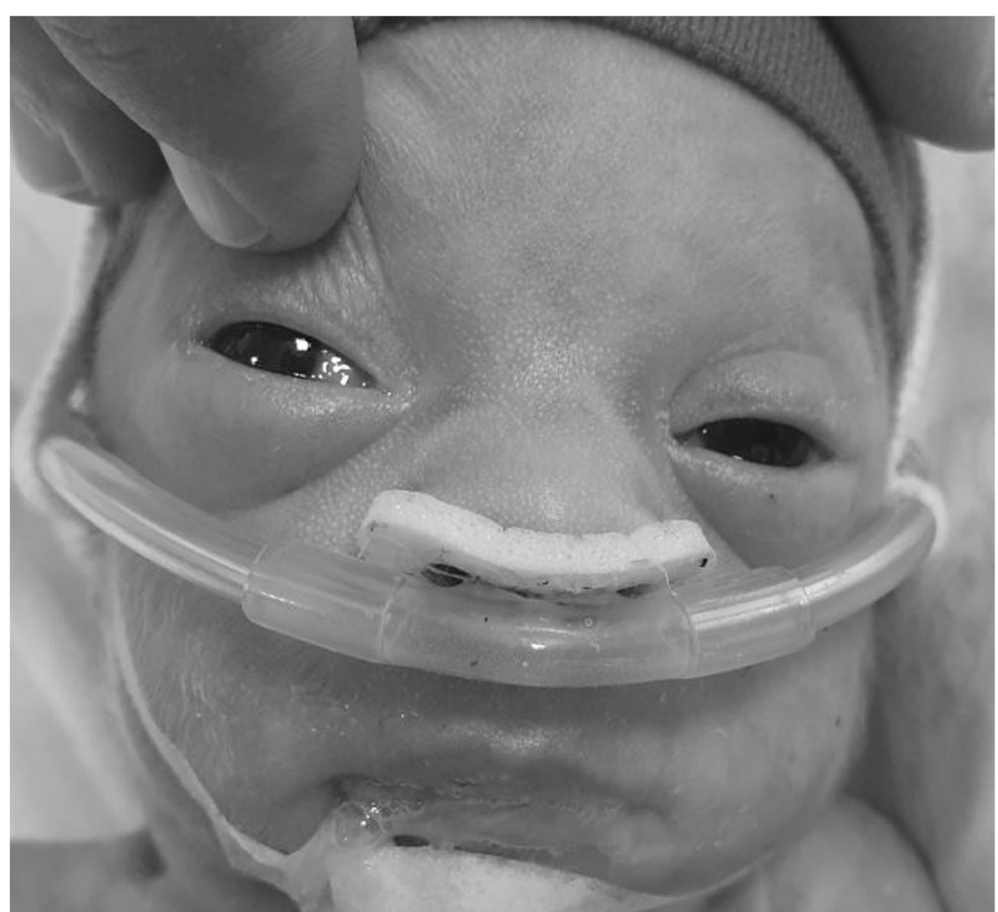

Fig. 1 Neonatal dysmorphic facial features: prominent forehead, hypertelorism, broad nasal bridge, flattened philtrum, thin upper lip. Right exophoria is present

potentials (VEP) recorded to binocular flash stimulation showed a delayed P100 latency $(240 \mathrm{msec}$ in right hemisphere, $270 \mathrm{msec}$ in left hemisphere), and brainstem auditory evoked potentials (BAEP) showed increased hearing threshold $(60 \mathrm{~dB})$ in the right ear and absence response even at high-intensity stimulation $(110 \mathrm{~dB})$ in the left ear. Extended newborn screening for inherited metabolic disorders and aCGH were negative. Thus, on the basis of the clinical evolution suggesting a mitochondrial encephalopathy, a further metabolic diagnostic work-up was carried on. Plasma amino acids, acylcarnitines and biotinidase were normal, while urinary organic acids analysis showed marked increase of 4-OH phenylacetic acid, along with a moderate increase of phenylpyruvic and mild increase of ethylmalonic and fumaric acids. Thus, at 5 months CA, WGS analysis was performed. The genomic sequencing,

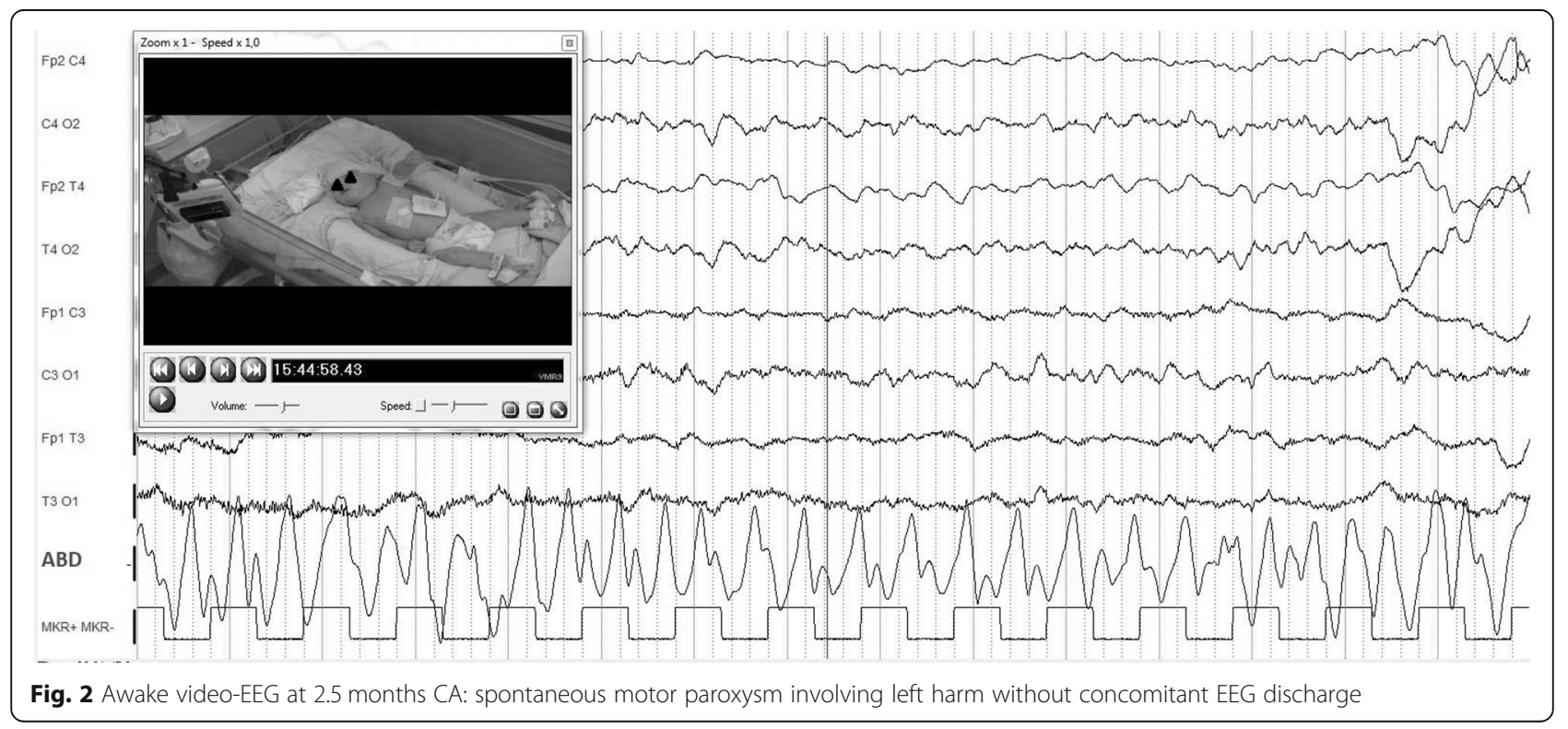




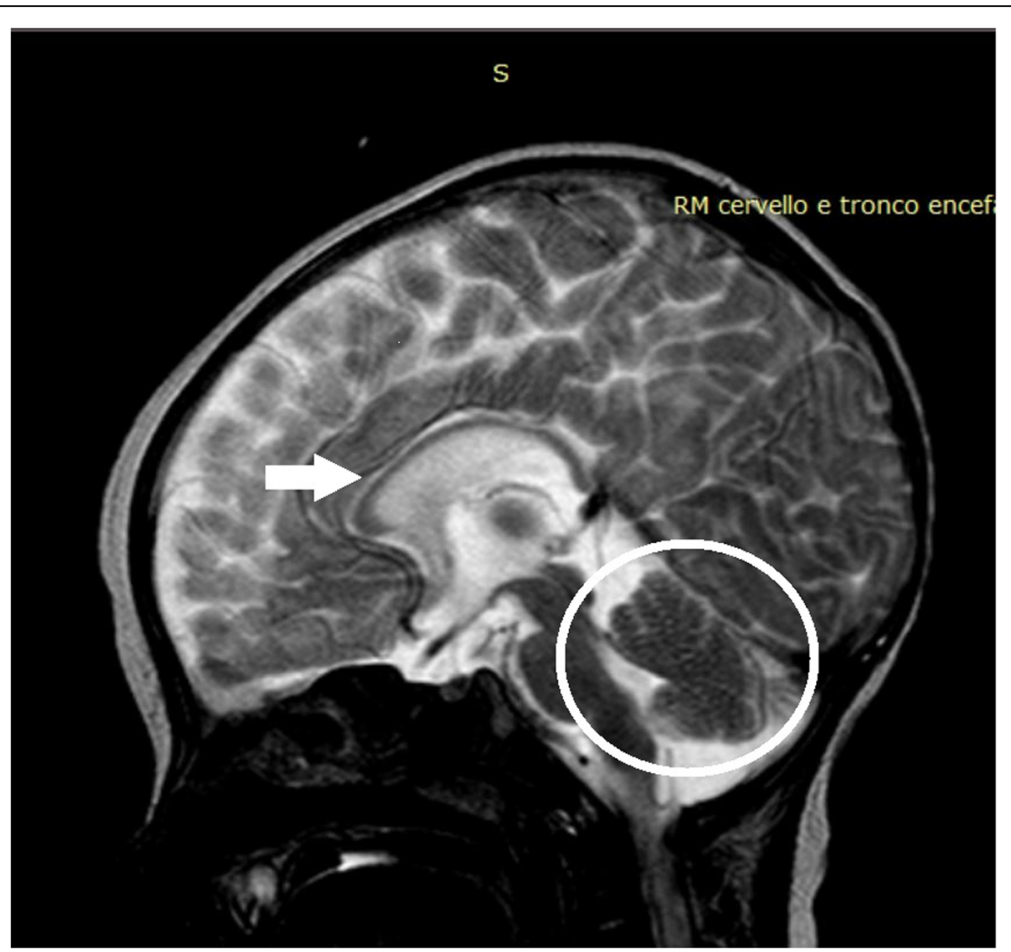

Fig. 3 Brain MRI T2 sagittal image: mild diffuse cerebral atrophy, hypoplasia of the corpus callosum (white arrow), cerebellar vermis hypoplasia (white circle)

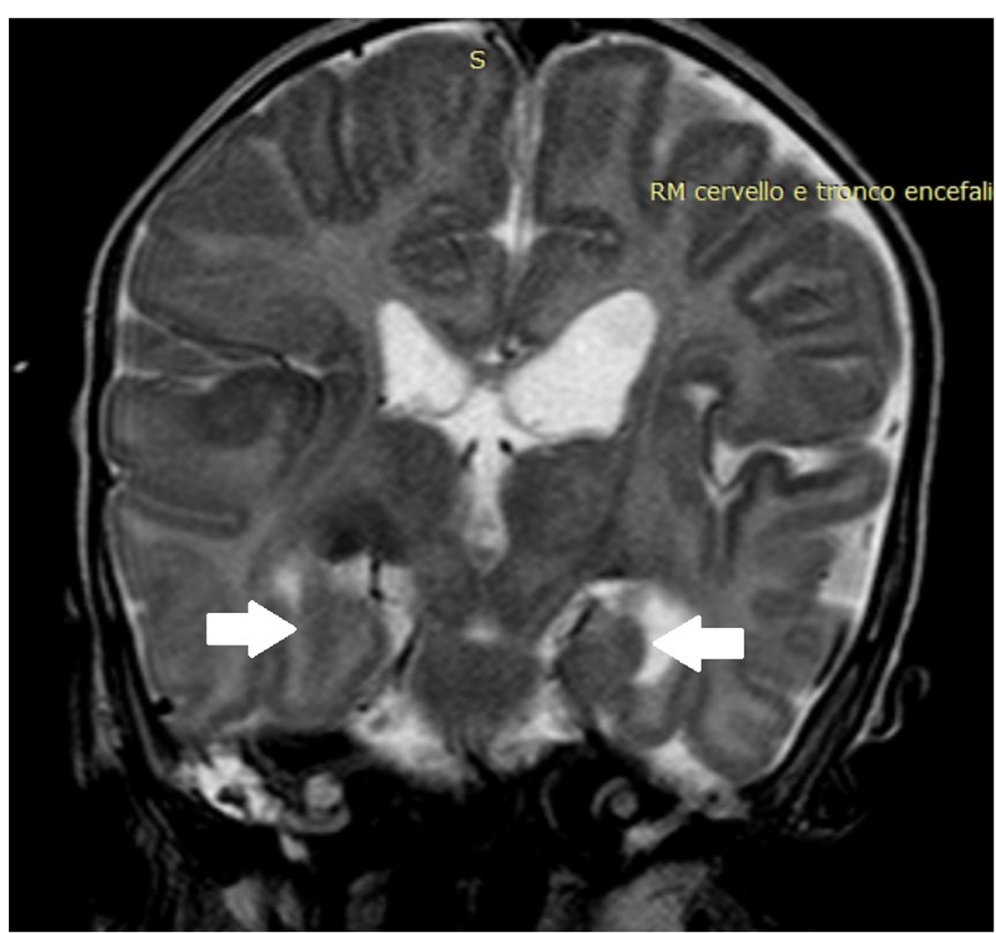

Fig. 4 Brain MRI T2 coronal image: incomplete bilateral hippocampal inversion (white arrows) and left lateral ventricle dilatation 
followed by direct sequencing, found and confirmed that the patient carried a compound heterozygous mutation in the LRPPRC gene, c.1921-7A $>\mathrm{G}$ and c.2056A $>\mathrm{G}$ (p.Ile686Val), splicing-site and missense variants, inherited from the mother and the father, respectively. The mutation of maternal origin and has not hitherto been described in the literature. Therefore, based on current knowledge, the variant can be classified as likely pathogenic (class IV). The second variant of paternal origin consisted in a replacement of an adenine with a guanine in position 2056 of the codon 686, determining the substitution of isoleucine with valine. This variant has not been described in the literature too, and according to current knowledge, can be classified of uncertain significance (class III). Parents refused any further invasive investigation for the execution of functional studies that could demonstrate COX activity deficit. Thus, to assess the functional effect of mRNA transcripts, we performed a reverse transcription-polymerase chain reaction (RT-PCR) that demonstrated the deletion of 45 base pairs in the exon 19 with a likely deletion of 15 amino acids in the encoded protein. He is currently 14 months CA, his weight is $5.500 \mathrm{~g}(-5.4 \mathrm{SD})$, length $65 \mathrm{~cm}(-5.2 \mathrm{SD})$ and OFC $40.6 \mathrm{~cm}(-4.6 \mathrm{SD})$. Owing to the severe generalized hypotonia he has to lie in bed with nasal cannula $\mathrm{O} 2$ supplementation. He shows only sporadic movements of head rotation without focusing on a nearby object. Neither vocalization nor blinking reaction to bell and rattle are present. He is unable to move his limbs and maintains adducted thumbs in both hands (Fig. 5). Nutritional supply with protein hydrolysate, MCT, Vitamin supplements, Lcarnitine and glycopyrrolate for drooling are provided by PEG. However, he has never experienced to date episodes of acute ketosis, glycemic derangements, or any acute stroke like events. A home rehabilitation treatment has been started.

\section{Discussion and conclusions}

In 1994, Hou first identified the LRPPRC gene, which encodes for a protein containing 35 amino acid repeat motifs [9], that conferring an ability to recognize RNA substrates, regulate a number of post-transcriptional processes such as RNA editing, stability or degradation [4]. Next generation sequencing, including WGS, Whole Genome Sequencing and RNA sequencing, has revolutionized the diagnostic approach of rare genetic disorders, making it possible to undertake a phenotyping driven by the results from genetic markers, called "reverse phenotyping" approach $[10,11]$.

In our patient we adopted a "reverse phenotyping" approach, since WGS analysis through the identification of novel LRPPRC compound heterozygous mutations, made it possible to refine the patient phenotype and the diagnosis of LSFC.

We identified a novel pathogenic compound heterozygous LRPPRC mutation in a male Italian child from Sicilian parents, consisting of a maternal splicing site (class IV) and a paternal missense (class III) variant. Thus, our proband did not carry, the most reported French-Canadian founder mutation. However, his clinical features resembled LSFC in many aspects including severe global neurodevelopmental impairment, generalized hypotonia, dystonia, dysphagia, exophoria, visual and hearing impairment and post-natal growth deficiency. In our patient brain anomalies and reduced neuronal cell proliferation leading to cortical atrophy, hypoplasia of corpus callosum, cerebellar vermis hypoplasia, left lateral ventricular dilatation,

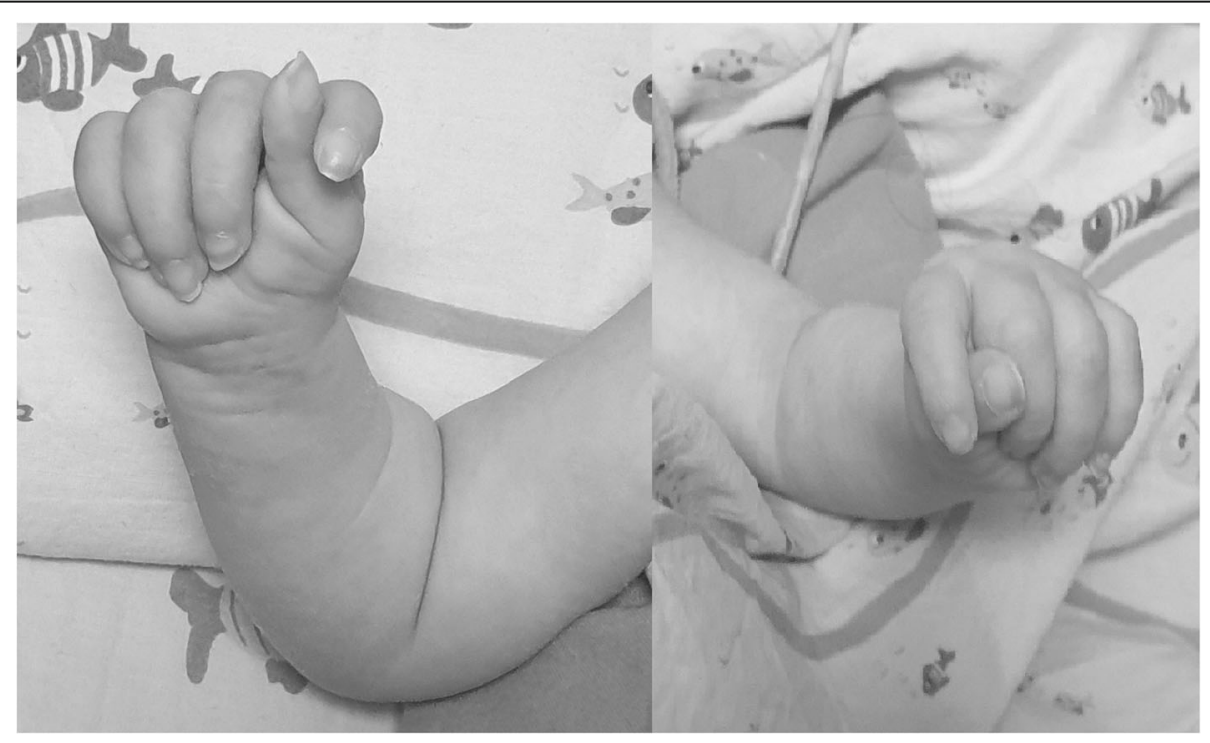

Fig. 5 Patient 14 months CA: Bilateral adducted thumbs 
unfolded hippocampi, have been evidenced by brain MRI. We suppose that the described involvement of basal ganglia, and midbrain, reported in some patients was not still present probably owing to the younger age [6]. Neither episodes of severe (sometimes fatal) lactic acidosis, nor ketosis or hyperglycemia were present in our patient, whose lactic acid levels maintained from birth levels $<2.5 \mathrm{mmol} / \mathrm{l}$. Urinary organic acids analysis showed marked increase of 4-OH phenylacetic acid, along with a moderate increase of phenylpyruvic and mild increase of ethylmalonic and fumaric acids. These results were not suggestive of mitochondrial deficit. Since in our patient we have not performed Western blot analyses for dosing protein levels of subunits of mitochondrial respiratory chain complexes, we can only speculate that a less severe compound heterozygous mutation could have avoided, to date, a fatal metabolic crisis, as well as the onset of left ventricle cardiomyopathy frequently found by others [6]. Indeed, these last deleterious effects have been found to be more frequently related to the LRPP $R C$ A354V founder mutation, responsible for an increased mitochondrial vulnerability and nutrient-induced cytotoxicity [12]. Moreover, different dose effects could also result from functional differences in gene expression depending on the parental origin of the single mutation, as recently described in other neurodevelopmental disorders [13].

A pathogenic link between LSFC and Neurofibromatosis type 1 (NF1), a clinically heterogeneous, neurocutaneous genetic disorder [14], has been recently found studying the RNA granules. The RNA granules play a key role in regulating de novo protein synthesis in a temporal and spatial manner by microtubule-dependent, motor proteindriven transport of mRNA cargoes to subcellular sites of requirement [15]. The tubulin binding domain of NF1 is a binding partner of the LRPPRC protein and both proteins complex with Kinesin 5B, Staufen1, hnRNP A2, and Myelin Basic Protein mRNA, likely in RNA granules. These findings provide clues to how loss or mutation of NF1 and $L R P P R C$ may contribute to the wide range of clinical manifestations reported in both condition [16]. Despite significant advances in understanding the molecular genetics of LSFC, the pathogenic mechanisms (precise molecular ones through which LRPPRC stabilizes mitochondrial transcripts) underlying this severe and unpredictable disease remain unclear. In most mitochondrial diseases, impaired capacity to generate ATP is believed to be the main culprit and mitochondria play a central role in other numerous vital processes (including triggering of programmed cell death), all of which could contribute to dysfunction and death, particularly when cells are faced with stressful conditions. On the other hand, a compensatory mechanism to preserve ATP level and consisting in the activation of the mTORC1 pathway and its downstream target HIF-1 $\alpha /$ PDHK1n LSFC fibroblasts has been recently described [17].
Effective treatment strategies for these patients are lacking. Recent progress achieved in pharmacogenomics might provide an effective treatment targeting potential consequences of COX deficiency such as impaired electron transport chains flux, oxidative stress, mitochondrial cell death signaling, and accumulation of toxic metabolites with interesting concerns about the nature of the diets, particularly excess fat intake, as well as on the use of antioxidants in patients with LSFC and, possibly, other COX defects have been raised. Indeed, Burelle et al. have demonstrate the protective effect of compounds (methylene blue and dinitrophenol) that promote flux through the electron transport chain independent of phosphorylation, and that modulate fatty acid (L-carnitine) or Krebs cycle metabolism (propionate), while antioxidants (resveratrol, idebenone, Nacetyl cysteine) exacerbate palmitate plus lactate-induced cell death [12].

In this report we have described the clinical and genetic aspects of a novel compound heterozygous mutation of $L R P P R C$ in an Italian male child with early onset LSFC characterized by severe neurologic involvement and facial dysmorphic features. The absence of early metabolic crisis or relevant lactic acidosis broaden the spectrum of phenotypes of LSFC. Our patient is one of the few reported outside of the French-Canadian population and the first, to our knowledge, observed in Italy. Integrated and family centered model of care for potential end-of-life pathologies should implement protocols that reduce unreasonable therapeutic obstinacy [18] and offer parents acceptance and commitment based coping strategies [19]. WGS analysis, a pivotal genetic test for early diagnosis, prognostic definition and accurate genetic counselling, should be performed in infants and children with hypotonia and developmental delay in whom metabolic investigations are normal and chromosomal aneuploidy, microdeletions and microduplications have been excluded by aCGH. Moreover, it has been recently proposed as a powerful tool for the diagnostic evaluation of critically ill infants with suspected monogenic disorders in the Neonatal and Pediatric Intensive Care Units and its use has a notable effect on clinical decision-making process [20].

\section{Supplementary information}

Supplementary information accompanies this paper at https://doi.org/10. 1186/s13052-020-00903-7.

Additional file 1.

\section{Abbreviations}

aCGH: Array Comparative Genomic Hybridization; LSFC: Leigh Syndrome French Canadian type; LRPPRC: Leucine-rich pentatricopeptide repeatcontaining; WGS: Whole-genome sequencing; COX: Cytochrome-c oxidase; CrA: Chronological age; CA: Corrected age for prematurity; OFC: Occipitofrontal circumference; US: Ultrasound; PEG: Percutaneous endoscopic gastrostomy; VEP: Visual evoked potentials; BAEP: Brain auditory 
evoked potentials; dB: Decibel; RT-PCR: Reverse transcription-polymerase chain reaction; SD: Standard deviation; NF1: Neurofibromatosis type1

\section{Acknowledgments}

Not applicable.

\section{Authors' contributions}

EP was primary involved in neonatal neurological and neurophysiological assessments. Performed brain US and neurodevelopmental follow-up and drafted the manuscript. GS was primary involved in collecting the current literature and drafting the manuscript. VA gave a substantial contribution for genetic testing MG was primary involved in acquisition of clinical data. EG performed WGS data confirmation by Sanger sequencing RNA analysis. FS was involved in submission to WGS analysis. IAMS performed clinical assessment and drafted the manuscript. $A B$ was primary involved in genetic testing and data management. GC supervised clinical as well as genetic assessment and revised the final manuscript. All authors approved the final manuscript as submitted and agree to be accountable for all aspects of the work.

\section{Funding}

The WGS analysis was carried out as part of a research project supported by Illumina "IHope" Network and Ministero dell'Istruzione, dell'Università e della Ricerca - MIUR "Dipartimenti di Eccellenza 2018-2022" to Department of Medical Sciences (Project D15D18000410001), University of Torino.

\section{Availability of data and materials}

The clinical data used during the current study are available from the corresponding author on reasonable request.

\section{Ethics approval and consent to participate}

This study was approved by the ethics committee Palermo 1 of "Paolo Giaccone" University Hospital of Palermo, Italy and parent's informed consent was provided.

\section{Consent for publication}

Parent's informed written consent was provided.

\section{Competing interests}

Not applicable.

\section{Author details}

'Department of Health Promotion, Mother and Child Care, Internal Medicine and Medical Specialties "G. D'Alessandro". University Hospital "P.Giaccone", University of Palermo, Piazza delle Cliniche, 2, 90127 Palermo, Italy. ${ }^{2}$ Department of Medical Sciences, University of Torino, Via Santena 19, 10126 Torino, Italy. ${ }^{3}$ Institute for Maternal and Child Health IRCCS Burlo Garofolo, Via dell' Istria, 65, 34137 Trieste, Italy.

\section{Received: 15 July 2020 Accepted: 16 September 2020}

Published online: 24 September 2020

\section{References}

1. Debray FG, Morin C, Janvier A, Villeneuve J, Maranda B, Laframboise R, Lacroix J, Jean-Claude Decarie JC, Yves Robitaille Y, Marie Lambert M, Brian $\mathrm{H}$, Robinson BH, Grant A, Mitchell GA. LRPPRC mutations cause a phenotypically distinct form of Leigh syndrome with cytochrome c oxidase deficiency. J Med Genet. 2011:48:183-9.

2. Merante F, Petrova-Benedict R, MacKay N, Mitchell G, Lambert M, Morin C, De Braekeleer M, Laframboise R, Gagne R. Robinson BH (1993) a biochemically distinct form of cytochrome oxidase (COX) deficiency in the Saguenay-lac-saint-Jean region of Quebec. Am J Hum Gen. 1993;53:481-7.

3. Morin C, Mitchell G, Larochelle J, Lambert M, Ogier H, Robinson BH, De Braekeleer M. Clinical, metabolic, and genetic aspects of cytochrome c oxidase deficiency in Saguenay-lac-saint-Jean. Am J Hum Genet. 1993;53:488-96.

4. Lightowlers RN, Chrzanowska-Lightowlers ZM. PPR (pentatricopeptide repeat) proteins in mammals: important aids to mitochondrial gene expression. Biochem J. 2008;416:e5-6.

5. Lightowlers RN, Chrzanowska-Lightowlers ZM. Human pentatricopeptide proteins: only a few and what do they do? RNA Biol. 2013;10:1433-8.
6. Sasarman F, Nishimura T, Antonicka H, Weraarpachai W, Shoubridge EA. Tissue-specific responses to the LRPPRC founder mutation in French Canadian Leigh syndrome. Hum Mol Genet. 2015;24:480-91.

7. Morin C, Dubé J, Robinson BH, Lacroix J, Michaud J, De Braekeleer M, Geoffroy G, Lortie A, Blanchette C, Lambert MA, Mitchell GA. Stroke-like episodes in autosomal recessive cytochrome oxidase deficiency. Ann Neurol. 1999;45(3):389-92.

8. Olahova M, Hardy SA, Hall J, Yarham JW, Haack TB, Wilson WC, Alston CL, He L, Aznauryan E, Brown RM, Brown GK, Morris AAM, Mundy H, Broomfield A, Barbosa IA, Simpson MA, Deshpande C, Moeslinger D, Koch J, Stettner GM, Bonnen PE, Prokisch H, Lightowlers RN, McFarland R, ChrzanowskaLightowlers ZMA, Taylor RW. LRPPRC mutations cause early-onset multisystem mitochondrial disease outside of the French-Canadian population. Brain. 2015;138:3503-19.

9. Hou J, Wang F, McKeehan WL. Molecular cloning and expression of the gene for a major leucine-rich protein from human hepatoblastoma cells (HepG2). In Vitro Cell Dev Biol Anim. 1994;30A:111-4.

10. Schulze TG, McMahon FJ. Defining the phenotype in human genetic studies: forward genetics and reverse Phenotyping. Hum Hered. 2004;58:131-8.

11. De Goede C, Yue WW, Yan G, Ariyaratnam S, Chandler KE, Downes L, Khan $\mathrm{N}$, Mohan M, Lowe M, Banka S. Role of reverse phenotyping in interpretation of next generation sequencing data and a review of INPP5E related disorders. Eur J Paediatr Neurol. 2016;20(2):286-95.

12. Burelle Y, Bemeur C, Rivard ME, Thompson Legault J, Boucher G, Morin C, LSFC Consortium, Coderre L, Des Rosiers C. Mitochondrial vulnerability and increased susceptibility to nutrient-induced cytotoxicity in fibroblasts from leigh syndrome French Canadian patients. PLoS One. 2015;10:e0120767.

13. Corsello G, Salzano E, Vecchio D, Antona V, Grasso M, Malacarne M, Carella M, Palumbo P, Piro E, Giuffrè M. Paternal uniparental disomy chromosome 14-like syndrome due a maternal de novo $160 \mathrm{~kb}$ deletion at the $14 \mathrm{q} 32.2$ region not encompassing the IG- and the MEG3-DMRs: patient report and genotype-phenotype correlation. Am J Med Genet A. 2015;167A(12):3130-8.

14. Corsello G, Antona V, Serra G, Zara F, Giambrone C, Lagalla L, Piccione M, Piro E. Clinical and molecular characterization of 112 single-center patients with Neurofibromatosis type 1. Ital J Pediatr. 2018;44(1):45.

15. Kanai Y, Dohmae N, Hirokawa N. Kinesin transports RNA: isolation and characterization of an RNA-transporting granule. Neuron. 2004:43:513-25.

16. Arun V, Wiley JC, Kaur H, Kaplan DR, Guha A. A novel Neurofibromin (NF1) interaction with the Leucine-rich Pentatricopeptide repeat motif-containing protein links Neurofibromatosis type 1 and the French-Canadian variant of Leigh's syndrome in a common molecular complex. J Neurosci Res. 2013;91(4):494-505.

17. Mukaneza Y, Cohen A, Rivard MÈ, Tardif J, Deschênes S, Ruiz M, LSFC Consortium, Laprise C, Des Rosiers C, Coderre L. mTORC1 is required for expression of $\angle R P P R C$ and cytochrome-c oxidase but not HIF-1a in Leigh syndrome French Canadian type patient fibroblasts. Am J Physiol Cell Physiol. 2019;317(1):C58-67.

18. Feudtner C, Nathanson PG. Pediatric palliative care and pediatric medical ethics: opportunities and challenges. Pediatrics. 2014;133(1 Suppl):s1-7.

19. Burke K, Muscara F, McCarthy M, Dimovski A, Hearps S, Anderson V, Walser R. Adapting acceptance and commitment therapy for parents of children with life-threatening illness: pilot study. Fam Syst Health. 2014;32(1):122-7.

20. Meng L, Pammi M, Saronwala A, Magoulas P, Ghazi AR, Vetrini F, Zhang J, He W, Dharmadhikari AV, Qu C, Ward P, Braxton A, Narayanan S, Ge X, Tokita MJ, Santiago-Sim T, Dai H, Chiang T, Smith H, Azamian MS, Robak L, Bostwick BL, Schaaf CP, Potocki L, Scaglia F, Bacino CA, Hanchard NA, Wangler MF, Scott D, Brown C, Hu J, Belmont JW, Burrage LC, Graham BH, Sutton VR, Craigen WJ, Plon SE, Lupski JR, Beaudet AL, Gibbs RA, Muzny DM, Miller MJ, Wang X, Leduc MS, Xiao R, Liu P, Shaw C, Walkiewicz M, Bi W, Xia F, Lee B, Eng CM, Yang Y, Lalani SR. Use of Exome Sequencing for Infants in Intensive Care Units: Ascertainment of Severe Single-Gene Disorders and Effect on Medical Management. JAMA Pediatr. 2017;171(12):e173438.

\section{Publisher's Note}

Springer Nature remains neutral with regard to jurisdictional claims in published maps and institutional affiliations. 